\title{
Effect of Temperature on Atomic Scattering Curve
}

\section{Haque $\mathbf{M M}^{1 *}$, Hasan $\mathbf{M M}^{1}$ and Islam $\mathbf{M M}^{2}$}

${ }^{1}$ Department of Mathematics, Northern University, Dhaka, Bangladesh

${ }^{2}$ Department of Chemistry, Northern University, Dhaka, Bangladesh

\begin{abstract}
We have calculated the temperature dependence of the atomic scattering curve for the elements $\mathrm{Si}, \mathrm{C}, \mathrm{Pt}$ and $\mathrm{Cu}$. At first we have drawn the general atomic scattering curve for the above elements. A quantity ' $\mathrm{f}$ ', the atomic scattering factor, is said to describe the 'efficiency' of scattering of a given atom in a given direction. The actual calculation of ' $f$ ' involves $\sin \theta$, rather than $\theta$, so that the net effect is that 'f' decreases as the quantity $(\sin \theta) / \lambda$ increases. Secondly, we have considered Debye temperature factor using Debye model. And we observe that for Debye approximation, the curve decreases a little bit.
\end{abstract}

Keywords: Scattering curve; Scattering factor; Temperature factor; Debye temperature; Debye model, MATLLAB software package

\section{Introduction}

The atomic scattering factor ' $f$ is defined as the ratio of amplitudes:

$$
f=\frac{\text { amplitude of the wave scattered by anatom }}{\text { amplitude of the wave scattered byone electron }}
$$

It is clear that $f=Z$ (atomic number) for any atom scattering in the forward direction [1]. As $\theta$ increases, the waves scattered by individual electrons become more and more out of phase and ' $f$ ' decreases [2]. The atomic scattering factor depends also on the wavelengths of the incident beam [3]. At a fixed value of $\theta$, ' $f$ ' will be smaller, the shorter the wavelength, leading to greater interference between the scattered beams. Calculated values of ' $f$ ' for various atoms and various values of $(\sin \theta) / \lambda$ are tabulated in the Tables $1-3$. We see that the curve begins at the atomic number and decreases to very low values for scattering in the backward direction ( $\theta$ near $90^{\circ}$ ) for very short wavelengths [4-8]. Again we observe that due to temperature factor, the general atomic scattering curve is affected for Debye temperature approximation. From this point of view, we have studied the temperature factor dependence of the atomic scattering curve for Debye temperature approximation [9-11].

\section{Thermal (temperature factor) effects on the atomic scattering} curve: (Debye approximation)

So far, a crystal has been considered as a collection of atoms located at fixed points in the lattice [12]. Actually, the atoms undergo thermal vibration about their mean positions even at the absolute zero of temperature, and the amplitude of this vibration increases as the temperature increases [13]. We need any quantitative information about the temperature factor $\mathrm{e}^{-\mathrm{M}}$, but it is convenient to describe the calculation here before considering other thermal effects. Formally, this effect is included by defining $f$ as the atomic scattering factor of an atom undergoing thermal vibration, $f_{0}$ as the same quantity for an atom at rest, and the two is related by [14]

$$
f=f_{0} \times e^{-M}
$$

The quantity $f_{0}$ is then the scattering factor as usually tabulated. Because the intensity of any line depends on $f^{2}$ calculated intensities must be multiplied by $e^{-M}$ to allow for thermal vibration [15]. The quantity $\mathrm{M}$ depends on both the amplitude $u$ of thermal vibration and the scattering angle $2 \theta$ :

$$
M=2 \pi^{2}\left(\frac{\bar{u}^{2}}{d^{2}}\right)=8 \pi^{2} u^{-2}\left(\frac{\sin \theta}{\lambda}\right)^{2}=B\left(\frac{\sin \theta}{\lambda}\right)^{2}
$$

Where, $\mathrm{u}^{-2}$ is the mean square displacement of the atom in a direction normal to the diffracting planes. The exact calculation of $\mathrm{u}^{-2}$ as a function of temperature is extremely difficult [16], which means that $M$ or $B$ is tough to determine accurately. Peter Debye has given the following expression:

$$
M=\frac{6 h^{2} T}{m k \Theta^{2}}\left[\varphi(x)+\frac{x}{4}\right]\left(\frac{\sin \theta}{\lambda}\right)^{2}, \text { (Debye approximation) }
$$

Where, $h$ is the Planck's constant, $T$ is the absolute temperature, $m$ is the mass of the vibrating atom, $k$ is the Boltzmann's constant, $\Theta$ is the Debye characteristic temperature of the substance in $\mathrm{K}, x=/ T$, and $\phi(x)$ is a function tabulated [17], along with values of $\Theta, m=A / N$ where $A$ =atomic weight and $N=$ Avogadro's number, the coefficient of the bracketed terms becomes, by inserting the numerical values of the constants in eqn. (3):

$$
\frac{6 h^{2} T}{m k \Theta^{2}}=\frac{6 \times\left(6.63 \times 10^{-34}\right)^{2} \times\left(6.023 \times 10^{23}\right) T}{A \Theta^{2} \times 10^{-20}}=\frac{1.15 \times 10^{4} T}{A \Theta^{2}}
$$

If $\lambda$ is in angstroms. In eqn. (3) is approximate and applies only to elements with cubic crystal structure.

So, ultimately in eqn. (3) reduces to,

$$
M=\frac{1.15 \times 10^{4} T}{A \Theta^{2}}\left[\varphi(x)+\frac{x}{4}\right]\left(\frac{\sin \theta}{\lambda}\right)^{2}
$$

Again, $\phi(x)$ can be expressed as:

$$
\varphi(x)+\frac{x}{4}=1+\frac{x^{2}}{36}-\frac{x^{4}}{3600}
$$

Thus, $\mathrm{M}$ can be written as,

*Corresponding author: Haque MM, Department of Mathematics, Northern University Bangladesh, Dhaka, Bangladesh, Tel: +8801749951559; E-mail: mhtmth3372@gmail.com

Received July 29, 2018; Accepted September 12, 2018; Published September 19, 2018

Citation: Haque MM, Hasan MM, Islam MM (2018) Effect of Temperature on Atomic Scattering Curve. J Appl Computat Math 7: 417. doi: 10.4172/21689679.1000417

Copyright: (c) 2018 Haque MM, et al. This is an open-access article distributed under the terms of the Creative Commons Attribution License, which permits unrestricted use, distribution, and reproduction in any medium, provided the original author and source are credited. 


$$
M=\frac{1.15 \times 10^{4} T}{A \Theta^{2}}\left[1+\frac{x^{2}}{36}-\frac{x^{4}}{3600}\right]\left(\frac{\sin \theta}{\lambda}\right)^{2}
$$

Finally, we get,

$$
f=f_{0} \exp \left[-\frac{1.15 \times 10^{4} T}{A \Theta^{2}}\left(1+\frac{x^{2}}{36}-\frac{x^{4}}{3600}\right)\left(\frac{\sin \theta}{\lambda}\right)^{2}\right]
$$

\section{Calculation of general scattering factor}

As seen by incident X-ray photons, a sample consists of a spatially inhomogeneous sea of electrons. In fact, quantum mechanics indicates that each electron is itself spread into a diffuse cloud of negative charge. The important quantity is the charge density $\rho$ which is normally expressed in electron units so that the quantity $\rho D v$ is the ratio of charge in volume $d v$ to the charge of one electron. Thus, for each electron $\int \rho d V=1$. The scattering from a single atom must take into account the phase differences for scattering from different "portions" of the atom, and $f_{0}$, the atomic scattering factor is made up of the contributions from individual electrons $f_{e}$ given by

$$
f_{e}=\int \exp \left[(2 \pi i / \lambda)\left(\underline{S}-\underline{S}_{0}\right) \cdot r\right] \rho d V
$$

Calculation of the precise values of $f_{0}=\sum\left(f_{e}\right)_{n}$ depends on the detailed knowledge of the charge distribution $\rho$.

While it may not be precisely true, assuming that the charge distribution of electrons in an atom is spherical and equals $\rho=\rho(r)$ is a very useful approximation and always a simple expression to be obtained for $f_{0}$. Using $\mathrm{k}=(4 \pi / \lambda) \sin \theta$ and $\left(\underline{S}-\underline{S}_{0}\right) \cdot r=2 r \sin \theta \cos \phi$ and

$$
\begin{aligned}
& f_{0}=\sum_{n}\left(f_{e}\right)_{n}=\sum_{n} \int_{r}^{\infty} \int_{\phi}^{\pi} \exp (i k r \cos \phi) \rho_{n}(r) 2 \pi r^{2} \sin \phi d \phi d r \\
& =\sum_{n} \int_{0}^{\infty} 4 \pi r^{2} \rho_{n}(r) \frac{\sin k r}{k r} d r
\end{aligned}
$$

We know that, at small angles, i.e., small $k$ or $(\sin \theta) / \lambda$, approaches unity. If $\mathrm{Z}$ is the number of electrons in an atom,

Then,

$$
\sum_{n} \int_{0}^{\infty} 4 \pi r^{2} \rho_{n}(r) d r=Z
$$

and $f_{0}$ approaches $\mathrm{Z}$ at all small values of $\sin \theta / \lambda$. Thus, the changes in electron density affect the amplitude of scattered $x$-radiation regardless of the ordering or lack of ordering of the scattering atoms.

\section{A. Calculation of atomic scattering factors for SI}

Using the proper charge distribution $\rho=\rho(\mathrm{r})$ of electrons in the atom of $\mathrm{Si}$ in eqn.(8), we readily obtain the atomic scattering factors for Si corresponding to $(\sin \theta) / \lambda$, below Table 1 .

B. Calculation for scattered atomic scattering factor (Debye approximation)

Here, the equation:

$$
f=f_{0} \times \exp \left[-\frac{1.15 \times 10^{4} T}{A \Theta^{2}}\left(1+\frac{x^{2}}{36}-\frac{x^{4}}{3600}\right)\left(\frac{\sin \theta}{\lambda}\right)^{2}\right]=f_{0} \times e^{-M}
$$

For Si, Atomic weight A=28.086, Debye characteristic Temperature $\Theta=645 \mathrm{~K}$, Absolute Temperature T in Kelvin $(100,150,200,250,300$, $350,400,450,500,550,600,650$ ).

Using the information obtained in the Tables 1-3, we plot the atomic scattering curve, giving $(\sin \theta) / \lambda$ in the $x$-direction and atomic scattering factors $f_{0}$ in the $y$-direction, we obtain the curve:

\section{Calculation of atomic scattering factors for $\mathrm{Cu}$}

Similarly, using the proper charge distribution $\rho=\rho(\mathrm{r})$ of electrons in the atom of $\mathrm{Cu}$ in eqn. (8), we readily obtain the atomic scattering factors for Si corresponding to $\sin \theta / \lambda$, below:

D. Calculation for scattered atomic scattering factor (Debye approximation)

Here, the equation:

$f=f_{0} \times \exp \left[-\frac{1.15 \times 10^{4} T}{A \Theta^{2}}\left(1+\frac{x^{2}}{36}-\frac{x^{4}}{3600}\right)\left(\frac{\sin \theta}{\lambda}\right)^{2}\right]=f_{0} \times e^{-M}$

For $\mathrm{Cu}$, Atomic weight $\mathrm{A}=63.546$, Debye characteristic Temperature $\Theta=343.5 \mathrm{~K}$, Absolute Temperature $\mathrm{T}$ in $\mathrm{K}(100,150,200$, $250,300,350,400,450,500,550,600,650,700)$.

Using the information obtained in the Tables $4-6$, we plot the atomic scattering curve, giving $(\sin \theta / \lambda)$ in the $x$-direction and atomic scattering factors $f_{0}$ in the $y$-direction, we obtain the curve.

\section{E. Calculation of atomic scattering factors for $\mathrm{Pt}$}

As above, from eqn. (8), we readily obtain the atomic scattering factors for Pt corresponding to $(\sin \theta) / \lambda$, below:

F. Calculation for scattered atomic scattering factor (Debye

\begin{tabular}{|c|c|c|c|c|c|c|c|c|c|c|c|c|}
\hline$(\sin \theta) / \lambda$ & 0.0 & 0.1 & 0.2 & 0.3 & 0.4 & 0.5 & 0.6 & 0.7 & 0.8 & 0.9 & 1.0 & 1.1 \\
\hline $\mathrm{Si}\left(f_{0}\right)$ & 14 & 11.35 & 9.4 & 8.2 & 7.15 & 6.1 & 5.1 & 4.2 & 3.4 & 2.95 & 2.6 & 2.3 \\
\hline \multicolumn{13}{|c|}{ Table 1: General Atomic Scattering Factors for Si. } \\
\hline $\exp (-M)$ & 1.0 & 0.9979 & 0.9901 & 0.9744 & 0.9484 & 0.9103 & 0.8592 & 0.7952 & 0.7195 & 0.6345 & 0.5438 & 0.4516 \\
\hline$f=f_{0} \times e^{-m}$ & 14 & 11.33 & 9.31 & 7.99 & 6.78 & 5.55 & 4.38 & 3.34 & 2.45 & 1.87 & 1.41 & 1.03 \\
\hline \multicolumn{13}{|c|}{ Table 2: When the Debye Approximation temperature factor is considered as $\left(e^{-M}\right)$} \\
\hline $\exp (-M)$ & 1.00 & 0.9958 & 0.9804 & 0.9494 & 0.8994 & 0.8287 & 0.7383 & 0.6324 & 0.5176 & 0.4025 & 0.2957 & 0.2039 \\
\hline$f=f_{0} \times e^{-2 M}$ & 14 & 11.30 & 9.22 & 7.79 & 6.43 & 5.06 & 3.77 & 2.66 & 1.76 & 1.19 & 0.77 & 0.47 \\
\hline
\end{tabular}
approximation)

Here, the equation:

$$
f=f_{0} \times \exp \left[-\frac{1.15 \times 10^{4} T}{A \Theta^{2}}\left(1+\frac{x^{2}}{36}-\frac{x^{4}}{3600}\right)\left(\frac{\sin \theta}{\lambda}\right)^{2}\right]=f_{0} \times e^{-M}
$$

For Pt, Atomic weight A=195.08, Debye characteristic Temperature $\Theta=240 \mathrm{~K}$, Absolute Temperature T in Kelvin $(100,150,200,250,300$, $350,400,450,500,550,600,650,700$ ). 
Citation: Haque MM, Hasan MM, Islam MM (2018) Effect of Temperature on Atomic Scattering Curve. J Appl Computat Math 7: 417. doi: 10.4172/21689679.1000417

Page 3 of 4

Using the information obtained in the Tables7-9, we plot the atomic scattering curve for $\mathrm{Pt}$, giving $(\sin \theta) / \lambda$ in the $x$-direction and atomic scattering factors $f_{0}$ in the $y$-direction, we obtain the curve:

\section{G. Calculation of atomic scattering factors for $\mathrm{C}$}

Similarly, in eqn. (8), we readily obtain the atomic scattering factors for $\mathrm{C}$ corresponding to $(\sin \theta) / \lambda$, below:

H. Calculation for scattered atomic scattering factor (Debye approximation)

Here, the equation:

$$
f=f_{0} \times \exp \left[-\frac{1.15 \times 10^{4} T}{A \Theta^{2}}\left(1+\frac{x^{2}}{36}-\frac{x^{4}}{3600}\right)\left(\frac{\sin \theta}{\lambda}\right)^{2}\right]=f_{0} \times e^{-M}
$$

For C, Atomic weight A=12.011, Debye characteristic Temperature $\Theta=2230 \mathrm{~K}$, Absolute Temperature T in Kelvin $(100,150,200,250,300$, $350,400,450,500,550,600,650)$.

Using the information obtained in the Tables $10-12$ we plot the atomic scattering curve for $\mathrm{C}$, giving $(\sin \theta) / \lambda$ in the $x$-direction and atomic scattering factors $f_{0}$ in the $y$-direction, we obtain the curve

\section{Results and Discussions}

Atomic scattering curve decreases with temperature factor. We have calculated the effect of temperature of the atomic scattering curve of the elements $\mathrm{Si}, \mathrm{Cu}, \mathrm{Pt}$ and $\mathrm{C}$. for the calculation, firstly we have considered the general scattering and then considered the temperature factors $\left(\mathrm{e}^{-\mathrm{M}}\right)$ and $\left(\mathrm{e}^{-2 \mathrm{M}}\right)$ and obtain the Figures 1-4, using Debye approximation where Debye temperature factor

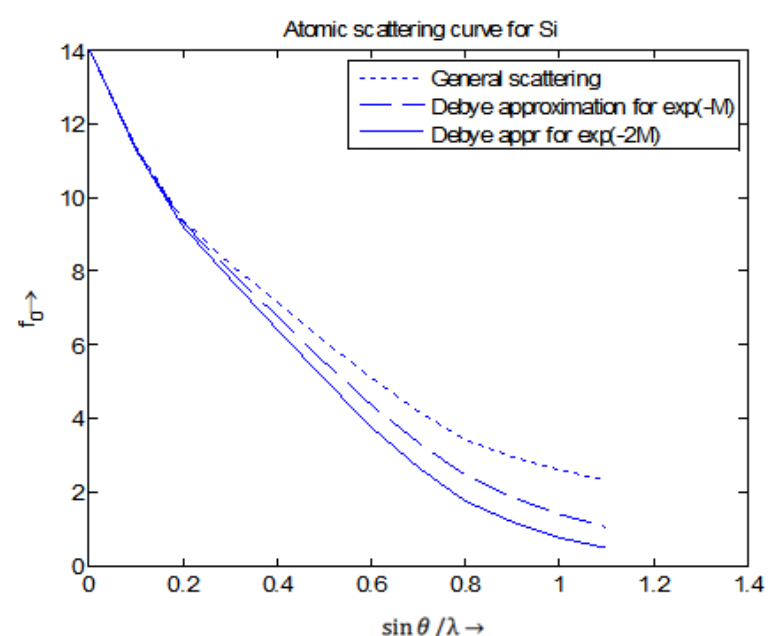

Figure 1: Temperature Dependence of Atomic scattering curve for Si with Debye Approximation.

\begin{tabular}{|c|c|c|c|c|c|c|c|c|c|c|c|c|c|}
\hline $\operatorname{Sin} \theta / \lambda$ & 0.0 & 0.1 & 0.2 & 0.3 & 0.4 & 0.5 & 0.6 & 0.7 & 0.8 & 0.9 & 1.0 & 1.1 & 1.2 \\
\hline $\mathrm{Cu}\left(f_{\partial}\right)$ & 29 & 25.9 & 21.6 & 17.9 & 15.2 & 13.3 & 11.7 & 10.2 & 9.1 & 8.1 & 7.3 & 6.6 & 6.0 \\
\hline \multicolumn{14}{|c|}{ Table 4: Atomic scattering for $\mathrm{Cu}$. } \\
\hline $\exp (-M)$ & 1.0000 & 0.9974 & 0.9868 & 0.9644 & 0.9266 & 0.8713 & 0.7982 & 0.7092 & 0.6082 & 0.5012 & 0.3951 & 0.2965 & 0.2109 \\
\hline$f=f_{0} \times e^{-M}$ & 29 & 25.83 & 21.31 & 17.26 & 14.08 & 11.58 & 9.34 & 7.23 & 5.53 & 4.05 & 2.88 & 1.96 & 1.27 \\
\hline \multicolumn{14}{|c|}{ Table 5: Debye approximation temperature factor is $\mathrm{e}^{-\mathrm{M}}$. } \\
\hline $\exp (-2 M)$ & 1.000 & 0.9948 & 0.9739 & 0.9300 & 0.8585 & 0.7592 & 0.6372 & 0.5029 & 0.3699 & 0.2512 & 0.1561 & 0.0879 & 0.0445 \\
\hline$f=f_{0} \times e^{-2 M}$ & 29 & 25.77 & 21.04 & 16.65 & 13.05 & 10.10 & 7.46 & 5.13 & 3.37 & 2.03 & 1.14 & 0.58 & 0.27 \\
\hline \multicolumn{14}{|c|}{ Table 6: Debye approximation temperature factor is $\mathrm{e}^{-2 \mathrm{M}}$. } \\
\hline $\operatorname{Sin} \theta / \lambda$ & 0.0 & 0.1 & 0.2 & 0.3 & 0.4 & 0.5 & 0.6 & 0.7 & 0.8 & 0.9 & 1.0 & 1.1 & 1.2 \\
\hline$P t\left(f_{\partial}\right)$ & 78 & 72.6 & 64.0 & 56.2 & 48.9 & 43.1 & 39.2 & 35.6 & 32.5 & 29.5 & 27.0 & 24.7 & 22.7 \\
\hline \multicolumn{14}{|c|}{ Table 7: Atomic scattering for Pt. } \\
\hline $\exp (-M)$ & 1.0000 & 0.9984 & 0.9915 & 0.9767 & 0.9512 & 0.9133 & 0.8617 & 0.7966 & 0.7192 & 0.6323 & 0.5397 & 0.4458 & 0.3552 \\
\hline $\begin{array}{c}f=f_{0} \times \\
\exp (-M)\end{array}$ & 78 & 72.48 & 63.46 & 54.89 & 46.51 & 39.36 & 33.79 & 28.36 & 23.37 & 18.65 & 14.57 & 11.01 & 8.06 \\
\hline
\end{tabular}

Table 8: Debye approximation temperature factor is considered as $\left(e^{-M}\right)$.

\begin{tabular}{|c|c|c|c|c|c|c|c|c|c|c|c|c|c|}
\hline $\exp (-2 M)$ & 1.0000 & 0.9967 & 0.9831 & 0.9539 & 0.9049 & 0.8341 & 0.7425 & 0.6345 & 0.5173 & 0.3998 & 0.2912 & 0.1987 & 0.1262 \\
\hline$f=f_{0} \times e^{-2 M}$ & 78 & 72.36 & 62.92 & 53.61 & 44.25 & 35.95 & 29.11 & 22.59 & 16.81 & 11.79 & 7.86 & 4.91 & 2.86 \\
\hline
\end{tabular}

Table 9: Debye approximation temperature factor is considered as $\left(e^{-2 M}\right)$.

\begin{tabular}{|c|c|c|c|c|c|c|c|c|c|c|c|c|c|}
\hline $\operatorname{Sin} \theta / \lambda$ & 0.0 & 0.1 & 0.2 & 0.3 & 0.4 & 0.5 & 0.6 & 0.7 & 0.8 & 0.9 & 1.0 & 1.1 & 1.2 \\
\hline$C\left(f_{0}\right)$ & 6 & 4.6 & 3.0 & 2.2 & 1.9 & 1.7 & 1.6 & 1.4 & 1.3 & 1.16 & 1.0 & 0.9 & 22.7 \\
\hline \multicolumn{14}{|c|}{ Table 10: Atomic scattering for $\mathrm{C}$. } \\
\hline $\exp (-M)$ & 1.0000 & 1.0019 & 0.9998 & 0.9937 & 0.9845 & 0.9723 & 0.9567 & 0.9377 & 0.9150 & 0.8882 & 0.8575 & 0.8227 & 0.3552 \\
\hline $\begin{array}{c}f=f_{0} \times \\
\exp (-M)\end{array}$ & 6 & 4.61 & 3.0 & 2.19 & 1.87 & 1.65 & 1.53 & 1.31 & 1.19 & 1.03 & 0.86 & 0.74 & 8.06 \\
\hline
\end{tabular}

Table 11: Debye Approximation temperature factor is considered as $\left(e^{-M}\right)$.

\begin{tabular}{|c|c|c|c|c|c|c|c|c|c|c|c|c|c|}
\hline $\exp (-2 M)$ & 1.0000 & 1.0015 & 0.9995 & 0.9875 & 0.9693 & 0.9453 & 0.9154 & 0.8793 & 0.8371 & 0.7890 & 0.7353 & 0.6769 & 0.1262 \\
\hline$f=f_{0} \times e^{-2 M}$ & 6 & 4.60 & 2.99 & 2.17 & 1.84 & 1.61 & 1.46 & 1.23 & 1.09 & 0.92 & 0.74 & 0.61 & 2.86 \\
\hline
\end{tabular}

Table 12: Debye approximation temperature factor is considered as $\left(e^{-2 M}\right)$. 
Citation: Haque MM, Hasan MM, Islam MM (2018) Effect of Temperature on Atomic Scattering Curve. J Appl Computat Math 7: 417. doi: 10.4172/21689679.1000417

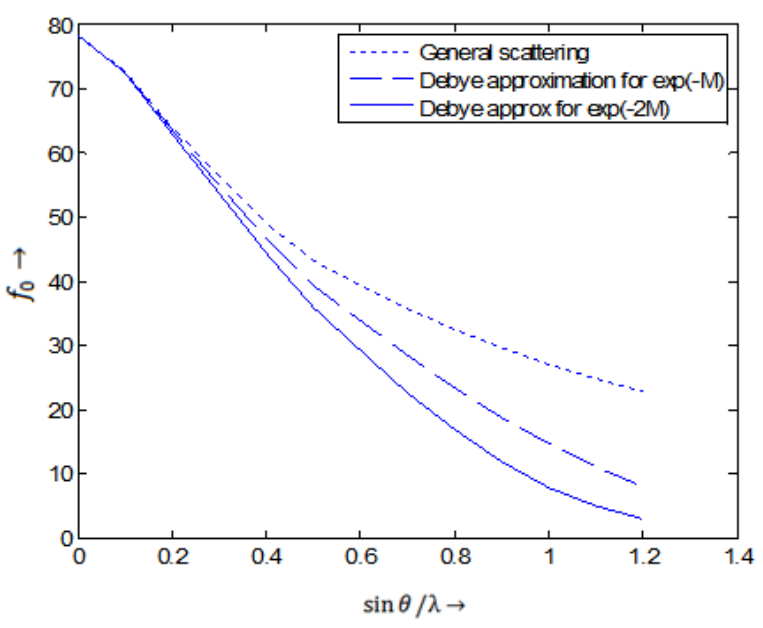

Figure 2: Temperature Dependence of Atomic scattering curve for Cu with Debye Approximation.

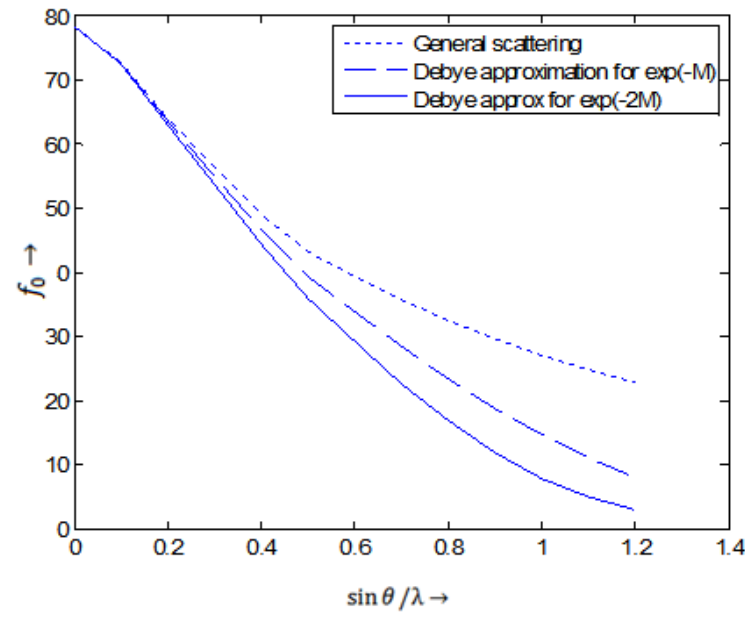

Figure 3: Temperature dependence of atomic scattering curve for Pt with Debye approximation.

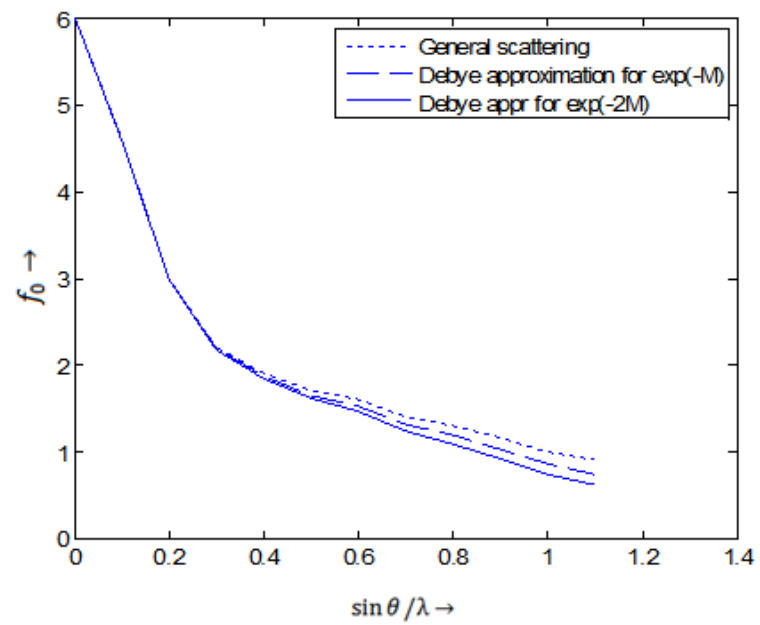

Figure 4: Temperature dependence of atomic scattering curve for $\mathrm{C}$ with Debye approximation. $\exp \left[-\frac{1.15 \times 10^{4} T}{A \Theta^{2}}\left(1+\frac{x^{2}}{36}-\frac{x^{4}}{3600}\right)\left(\frac{\sin \theta}{\lambda}\right)^{2}\right]$ and observe that the atomic scattering curve decreases a little bit compared with general scattering curve. So, atomic scattering curve is affected by the temperature factor.

\section{References}

1. Cullity BD, Stock SR (2014) Elements of X-ray Diffraction. Pearson Education

2. Mahmud MR, Rahman MO (2010) Temperature dependence of the reflection lines. Jahangirnagar University Journal of Science 3: 23-32.3.

3. Zachariasen WH (2004) Theory of X-ray Diffraction in Crystals. Courier Corporation.

4. Kittel C (1976) Introduction to solid state physics. New York: Wiley 8.

5. Chang SL, Höche HR (1985) Multiple diffraction of X-rays in crystals. Springer Series in Solid-State Sciences 50.

6. Obaidur RM (2002) Design, fabrication and performance of silicon monolithic monochromators and its application for lattice spacing measurements of $\mathrm{Si}$ and $\mathrm{GaAs}$ single crystals with synchrotron radiation.

7. Maidul IM (2014) Study of the Dynamical Theory of X-ray Diffraction with Absorption. Jahangirnagar University.

8. Authier A (2004) Dynamical theory of X-ray diffraction. Oxford University Press on Demand 11.

9. Warren BE (1990) X-ray Diffraction. Courier Corporation.

10. Jahirul I (2011) Study of the Dynamical Theory of X-ray Diffraction.

11. Bragg WL (1929) The diffraction of short electromagnetic waves by a crystal.

12. Seeger A (1962) Solid state physics. Zeitschrift für Physikalische Chemie 32 402-402.

13. Sah CT (1991) Fundamentals of solid state electronics. World Scientific Publishing Company.

14. James R (1948) The Optical Principles of the Diffraction of X-rays (Bell, London).

15. Bragg L (1968) X-ray crystallography. Scientific American 219: 58-74.

16. Reitz JR, Milford FJ, Christy RW (2008) Foundations of electromagnetic theory. Addison-Wesley Publishing Company.

17. Omar MA (1975) Elementary solid state physics: principles and applications. Pearson Education India. 